\title{
Integración de un curso MOOC y de un PLN-PLE en un curso presencial sobre fundamentos de la programación
}

\section{Integration of a MOOC course and a PLN-PLE in a face-to-face course on fundamentals of programming}

\author{
Joel Ricardo Jiménez Cruz \\ Universidad Autónoma Metropolitana-Iztapalapa, México \\ jcjr@xanum.uam.mx
}

\begin{abstract}
Resumen
En este trabajo se describen las experiencias educativas en un curso sobre Fundamentos de Programación en computadoras para alumnos de Ciencias Sociales y Humanidades que integra clases presenciales con elementos de la educación virtual como la participación en un curso masivo y abierto en línea (MOOC) y la elaboración de entornos y redes personales de aprendizaje (PLE-PLN). Esta hibridación de componentes presenciales y virtuales requiere de una integración y sincronización adecuada. En este sentido se ha tomado al curso MOOC como un "ayudante" en la impartición de la parte práctica del curso presencial y en el salón de clases se revisa la parte teórica y se llevan a cabo ejercicios y comentarios que tratan de integrar a las dos modalidades educativas. Esta integración se ve reforzada por el PLN-PLE que cada uno de los estudiantes va desarrollando conforme al avance en clase y en el curso masivo. Los PLNs-PLEs en su conjunto se pueden considerar como ciber-espacios de intercambio de información y construcción del conocimiento. La coordinación central de las actividades de ambas modalidades educativas se realiza por medio de un entorno virtual de enseñanza aprendizaje (EVEA) implementado en Moodle. El diseño instruccional que se ha seguido en clases está sustentado por la metodología del aprendizaje basado en proyectos que le permite al profesor y a los alumnos tener una visión y un objetivo bien planteados desde el principio del ciclo escolar. El marco pedagógico que soporta a los elementos presenciales y virtuales se da dentro de un contexto de aprendizaje conectivista que pretende alcanzar diferentes niveles de interacción e involucramiento cognitivo entre los participantes.
\end{abstract}

Palabras clave

MOOC, PLE, PLN, Diseño Instruccional, Conectivismo, B-learning.

\begin{abstract}
This paper describes the educational experiences in Computer Programming Fundamentals for students of Social Sciences and Humanities that integrates face-to-face classes with elements of virtual education such as participation in a Massive and Open Online Course (MOOC) and the development of personal learning environments and networks (PLEPLN). This hybridization of face-to-face and virtual components requires proper integration and synchronization. In this sense the MOOC course has been taken as an "assistant" in the delivery of a practical part of the course and in the classroom the theoretical part is reviewed and exercises and comments are carried out trying to integrate the two educational modalities. This integration is reinforced by the PLN-PLE that each student develops according to the progress achieved in class and in the massive course. PLNs-PLEs as a whole can be considered as cyber-spaces of information exchange and knowledge construction. The central coordination of the activities of both educational modalities is carried out in the classroom through a virtual learning and teaching environment (EVEA) implemented in Moodle. The instructional design that has been followed in classes is supported by the methodology of project-based learning that allows the teacher and the students to have a well-planned vision and objective from the beginning of the school
\end{abstract}


period. The pedagogical framework that supports the presence and virtual elements is given within a context of connectivist learning that seeks to achieve different levels of interaction and cognitive involvement among the participants.

Key words

MOOC, PLE, PLN, Instructional Design, Connectivism, B-learning.

\section{Introducción}

Las tecnologías en línea habilitan la emergencia de innovaciones disruptivas y cambios de paradigmas en la educación que responden a las exigencias económicas, sociales, culturales y políticas de la sociedad actual. Entre los ejemplos de estas emergencias se encuentran la educación semipresencial o híbrida y los cursos masivos abiertos y en línea (MOOCs) que son cursos que nacen con la idea de ser gratuitos y de que la educación llegue a todas las personas. La educación híbrida conjunta las bondades de la educación presencial y de la educación virtual.

En este trabajo, se presentan las experiencias de incluir en las clases presenciales la elaboración de un PLE-PLN por cada uno de los estudiantes y la participación en un curso MOOC. Esta inclusión tiene los propósitos de fomentar el aprendizaje centrado en el estudiante; complementar las competencias digitales necesarias hoy en día y homogenizar y elevar el nivel de los estudiantes nacionales al nivel de un estudiante de cualquier parte del mundo.

De acuerdo con Howard Rheingold (2010), los educadores debemos descubrir cómo involucrar a los estudiantes y a nosotros mismos, para ir más allá de las habilidades y las tecnologías, explorando las alfabetizaciones de medios sociales interconectados; (1) atención; (2) participación; (3) cooperación; (4) conciencia de la red; y (5) consumo crítico. Los medios sociales permiten dar forma a los entornos cognitivos, sociales y culturales de este siglo.

En las secciones que siguen se describe el contexto en el cual se desarrolló el presente trabajo, se da el sustento teórico a la parte presencia y a la parte virtual. Se explica la metodología que se utilizó y los resultados obtenidos y finalmente se comenta y discute el impacto de la combinación de los componentes presenciales y virtuales que requieren de una integración y sincronización adecuada.

\subsection{Contexto}

En este trabajo se describen las experiencias educativas surgidas en un curso presencial llamado "Fundamentos en Programación" que se ha impartido durante varios trimestres en la División de Ciencias Sociales y Humanidades (CSH) de la Universidad Autónoma Metropolitana Campus Iztapalapa, México. El curso está dedicado al estudio de los conceptos preliminares sobre computación y programación y tiene asignado un total de 4 horas a la semana durante 11 semanas que dura el trimestre.

Para la enseñanza de la programación se utiliza el lenguaje Scratch (https://scratch.mit.edu/). Este lenguaje se estudia en el salón de clases y a través del curso MOOC de la plataforma Coursera llamado "¡A programar!, una introducción a la programación” (Friss de Kereki, 2016). En este curso se recrean los ejercicios propuestos de Scracth y en el salón de clases se revisa la problematización algorítmica

Integración de un curso MOOC y de un PLN-PLE en un curso presencial sobre fundamentos de la programación. Joel Ricardo Jiménez Cruz. 
referente a la creación de pseudocódigos y diagramas de flujo, cuidando el proceso de integración de las dos modalidades educativas.

A medida que avanza el curso, los estudiantes van construyendo paulatinamente su PLE-PLN utilizando la aplicación para la elaboración de sitios web que proporciona la compañía WIX (http://es.wix.com/) en forma gratuita. Wix es una plataforma de desarrollo web, basada en el sistema de cómputo en la nube, que permite crear fácil y rápidamente una presencia online profesional, sin saber programar.

Como tarea inicial cada estudiante crea un blog personal con el fin de llevar una bitácora del curso y crear una plataforma social. Los blogs en su conjunto se pueden considerar como un foro en el cual se intercambia información y conocimiento. Posteriormente, los estudiantes diseñan e implementa en su mismo sitio de WIX una escuela virtual para la enseñanza de Scratch, escogiendo una población objetivo de niños, jóvenes o adultos.

La coordinación del curso presencial que dicta el profesor; el curso MOOC que llevan los estudiantes y los PLEs y PLNs que construyen ellos mismos, se lleva a cabo por un EVEA (Entorno Virtual de enseñanza aprendizaje) implementado en Moodle. En su conjunto todos los elementos empleados forman un ecosistema híbrido de enseñanza aprendizaje (figura 1). En la siguiente sección del marco teórico, se elucidan las concepciones teóricas y prácticas de estos componentes y las relaciones entre ellos.

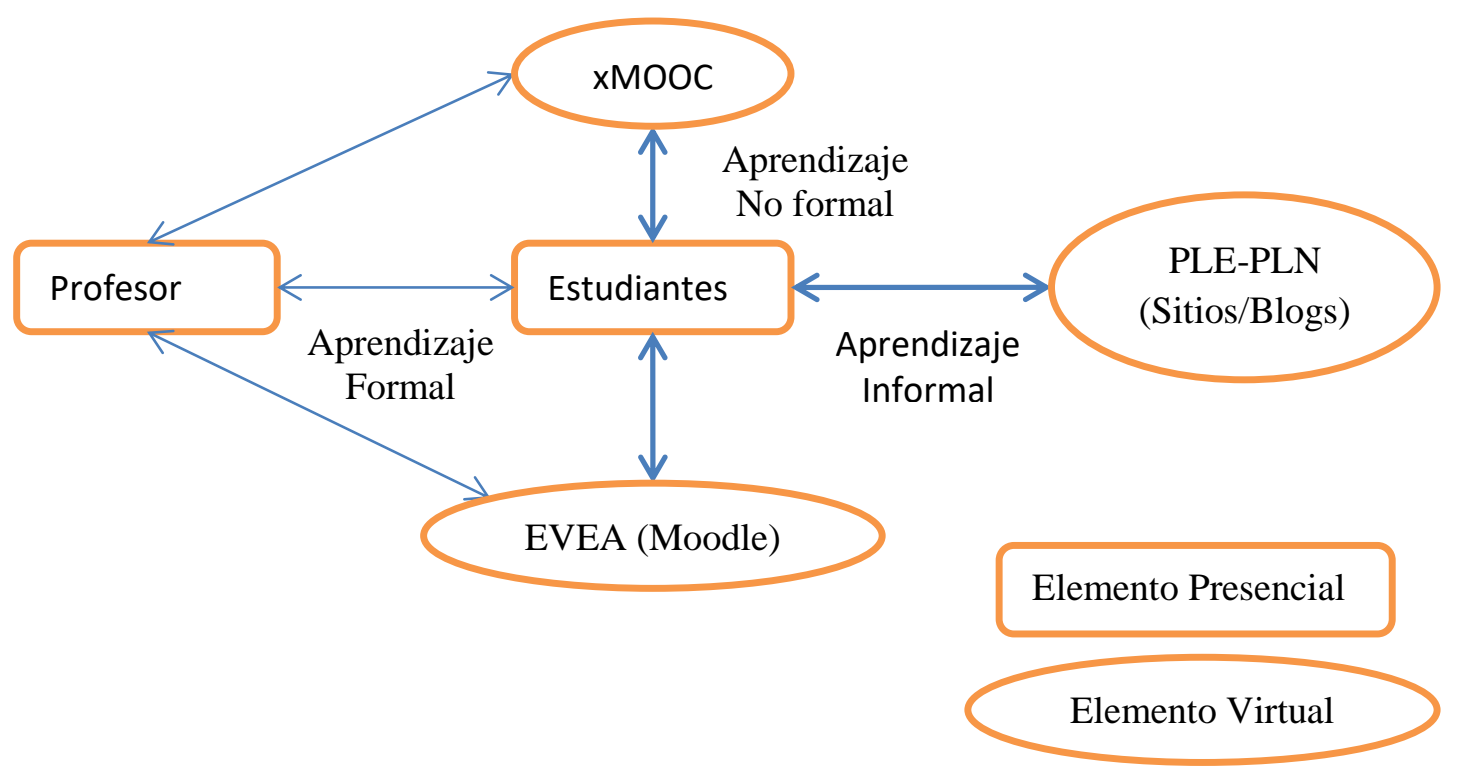

Figura 1. Modelo de un ecosistema híbrido de aprendizaje-enseñanza (Fuente: elaboración propia)

\section{Marco teórico}

El ecosistema híbrido, que se describe en este trabajo, representa un ciber-espacio conectivista donde la información y el conocimiento se haya distribuido entre los nodos 
y relaciones en una red (Siemens, 2004; Downes, 2009) y es adaptable a las necesidades tecno-pedagógicas sociales del trinomio universidad, estudiantes y profesor que permite crear un puente entre diferentes formas de aprendizaje-enseñanza dentro y fuera de la escuela; es decir, permite integrar y experimentar con los aprendizajes formales (presencial, dentro de la universidad, con apoyo de un EVEA); no formales (fuera de la universidad, en una institución, como la instrucción en un curso MOOC); e informales (fuera de la universidad, en cualquier lugar, por ejemplo; el aprendizaje a través de un PLE-PLN), (Eshach, 2007).

\subsection{Los PLE-PLN y los EVEA}

Los PLE-PLN son medios colaborativos de aprendizaje social que están conformados por recursos materiales, humanos y tecnológicos y funciones que puede utilizar o desarrollar una persona para aprender y/o enseñar un domino específico del conocimiento y que es extensible para toda la vida. Las funciones pueden ser: la gestión de la información y del conocimiento, la creación de contenidos y la conexión y participación en espacios similares con otros. Los recursos y funciones, permiten a los alumnos, tomar el control y gestionar su aprendizaje en función de la definición de sus propios procesos, objetivos, relaciones y comunicación con otros (Marín, Negre, Pérez, 2014).

Cada alumno a través de su PLE se relaciona con los PLEs de sus compañeros o de otras personas formando un PLN que permiten establecer lazos de comunicación y aprendizaje y en conjunto forman ecologías de conocimiento, cuya interacción recíproca da como resultado el surgimiento de estrategias que van incorporándose a su PLE y que van contribuyendo a la generación y distribución del conocimiento en la red de conexiones. Estos conceptos soportan una de las ideas centrales del conectivismo de que el aprendizaje y el conocimiento emergen por la habilidad de construir estas redes y de navegar por ellas (Downes, 2009).

Por otro lado, el EVEA funciona como un centro virtual de enseñanza que organiza diferentes actividades de acceso y consulta de información (guías, ejemplos, tareas, exámenes, actividades), crea espacios de comunicación entre los alumnos y el profesor y coordina la elaboración de los PLE-PLN de cada uno de los alumnos.

\subsection{Los cursos MOOC}

Los cursos MOOCs son fenómenos emergentes que incorporan varias concepciones de estos tiempos, entre ellas: un nuevo paradigma educativo; una tendencia educativa; una innovación disruptiva; una experimentación de la nueva educación; una punta de lanza de la educación que señala posibles caminos de evolución y que tienen el potencial de revolucionar y transformar la educación (Best College Online, 2013).

Los cursos MOOC son cursos virtuales en línea, accesible por Internet al que se puede apuntar cualquier persona y prácticamente no tiene límite de participantes. Son de carácter abierto y gratuito (Plourde, 2013). El éxito social de estos cursos se debe a la apertura de una educación universal para todos y la posibilidad de una interacción social con estudiantes de todo el mundo.

Integración de un curso MOOC y de un PLN-PLE en un curso presencial sobre fundamentos de la programación. Joel Ricardo Jiménez Cruz. 
Estas ideas señalan posibles cambios en el quehacer de las universidades, la acreditación académica, el mejoramiento en la calidad de la educación y su democratización.

Las intenciones de estos cursos también albergan las opiniones de democratización educativa de Ivan Illich (1985) que promueven el derecho a aprender a cualquier persona de una manera gratuita, casual y fuera de la escolarización, y fomentan el autoaprendizaje y la educación auto dirigida que son apoyadas por relaciones sociales libremente intencionadas y por encuentros y conversaciones fluidas e informales.

Según Scharmer (2015) ha habido una evolución de los MOOCs. En la generación 1.0 la comunicación era unidireccional; una persona hablaba y las demás escuchaban. Había contenido que se podía descargar. En la generación 2.0, se da la posibilidad de una mayor conversación y discusión entre los participantes. El estudiante reproduce el contenido y resuelve problemas. En la generación 3.0 se genera un mayor diálogo y una interacción autoreflexiva y multilateral. En la generación 4.0 se potencia la conversación y la recepción de retroalimentación por parte de los demás y emerge una inteligencia colectiva que promueve la innovación y la creatividad. La conversación se experimenta como un flujo de cocreación que permite a los participantes comunicar sus experiencias.

Los cursos MOOCs tienen varios desafíos que enfrentar como; los cuestionamientos sobre su validez como modelos de una trasformación educativa respecto a su alta masificación y el tratamiento y apoyo que se le da al alumno, sin considerar la individualización y la diversidad; los altos grados de deserción que tienen; la dificultad para verificar la identidad de los participantes; las acreditaciones y la ausencia de un enfoque pedagógico apropiado que incluya; actividad tutorial, evaluación, experiencia del usuario, recursos adecuados, planeación, administración y motivación. Según Hansen y Reich (2015), los participantes de los cursos MOOCs viven en colonias prósperas y educadas. Por otro lado, Dunbar (1993) menciona que existe un límite cognitivo para el número de personas (150) con el cual un individuo puede establecer y mantener relaciones estables.

Una categorización de los principales tipos de MOOCs que está basada en su estructuración y las diferentes experiencias educativas es la siguiente (Martí, 2012; Lane, 2012); los cMOOCs, en los cuales el aprendizaje se encuentran distribuidos en los nodos de la red y el conocimiento se construyen en forma global (teoría conectivista); los tMOOCs que están basados en la consecución de la resolución práctica de tareas o proyectos (instructivismo y constructivismo) y los xMOOCs que se encuentran basados en los contenidos y en una evaluación muy parecida a las clases tradicionales, con pruebas estandarizadas y automatizadas (conductivismo). En todos los MOOCs, sin embargo, se encuentran incorporados en mayor o en menor medida, los tres elementos de redes, tareas y contenidos.

Crosslin (2016) analiza los objetivos de un MOOC para determinar si el enfoque epistemológico instruccional debiera ser conectivista, instructivista o una mezcla de

Integración de un curso MOOC y de un PLN-PLE en un curso presencial sobre fundamentos de la programación. Joel Ricardo Jiménez Cruz. 
ambos. Cada enfoque considera la dinámica de poder y el diseño teórico que relacionan diversas actividades y expectativas en el curso.

Además de los materiales de un curso tradicional, como son vídeos, lecturas y cuestionarios, la inclusión de un curso MOOC dentro del esquema de la educación híbrida tiene varias ventajas; puede servir como material de estudio, como texto, audio o recursos multimedia; como "ayudante" del profesor; como plataforma de inducción al mundo digital y de relación con otros estudiantes en otras partes del mundo. Los MOOC proporcionan foros interactivos que ayudan a construir una comunidad virtual (Fernández, 2015).

Maria Joseph (2015) incluyó un curso MOOC en su curso presencial y encontró impactos positivos modestos en los resultados del aprendizaje, no encontró evidencia de un efecto negativo, pero si encontró bajos niveles de satisfacción en los estudiantes. Ella comenta los desafíos con los que se va enfrentando el profesor respecto a la integración y sincronización de los MOOCs con la clase. Menciona que estos cursos tienen el potencial de ofrecer recursos excelentes como las clases en videos, las evaluaciones y las tareas.

Bralić y Divjak (2016) describen la investigación sobre la incorporación de un curso MOOC en un curso tradicional. Sus objetivos se centraban en proporcionar la oportunidad de experimentar el aprendizaje virtual y ayudar a los estudiantes de tiempo parcial en su aprendizaje. Se pidió a los estudiantes que llevaran un diario de su aprendizaje con el doble propósito de evidenciar la comprensión del contenido de la materia y poder emitir una evaluación cualitativa y cuantitativa de la misma. El análisis cualitativo mostró un aprendizaje positivo. Los estudiantes se mostraron satisfechos a pesar de algunas dificultades, tales como la barrera del idioma, los conocimientos previos insuficientes o la carga de trabajo requerida. Las características más apreciadas de los MOOC fueron el aprendizaje a su propio ritmo y la opción de evaluar el conocimiento adquirido en los estudiantes de tiempo parcial.

Castaño, Garay y Maiz (2017) obtienen evidencia de que la participación activa en un curso MOOC y el diseño del curso presencial que utiliza las redes sociales y la realización de e-actividades mejora los resultados de aprendizaje en el curso.

En un curso de Programación de Computadoras y de microprocesadores para estudiantes universitarios de primer y segundo año, respectivamente, se integró un curso MOOC de Edx. En esos cursos se inscriben varios cientos de estudiantes de 3 campos diferentes del Instituto de Tecnología de Birla en India. Se pensó que la implantación de este modelo combinado reduciría la carga de trabajo de los profesores, pero no fue así. Sus resultados indican que los estudiantes con experiencia previa en programación se beneficiaron más de este modelo que los estudiantes irregulares que presentaban ausentismo y no aprendieron pese a tener los contenidos de la materia todo el tiempo (Tathagata, Malapati, Murthy, 2016).

Otra experiencia sobre la inclusión de un curso MOOC en el salón de clases, que se asemeja a una clase invertida (flipped-classrom), la reporta Salinas et al (2015). En esta

Integración de un curso MOOC y de un PLN-PLE en un curso presencial sobre fundamentos de la programación. Joel Ricardo Jiménez Cruz. 
experiencia, se deja a los estudiantes que estudien los videos para que en la clase se profundice el aprendizaje de los videos. Un objetivo central de su estudio fue el diseño de una forma de autoevaluación de los estudiantes. La calificación final del curso se calculó con el promedio de las catorce calificaciones semanales. La calificación de cada semana estuvo formada por $70 \%$ de la calificación de desempeño en el aula y $30 \%$ de la calificación de autoevaluación en Coursera. Los estudiantes vivieron con asombro la oportunidad para construir su propia calificación a medida que avanzaba el semestre. Se observó que este tipo de evaluación vuelve a los estudiantes menos dependientes del profesor y se hacen más responsables y autónomos.

A continuación se relatan las experiencias propias surgidas con la incorporación de un curso MOOC en el curso presencial de Fundamentos de la Programación.

\subsection{La inclusión en clase de un curso MOOC de la plataforma Coursera}

En la materia presencial "Fundamentos de programación" se incluyó el curso MOOC de Coursera "¡A programar!, una introducción a la programación” (figura 2). Este curso promueve el pensamiento sistémico al presentar la descomposición de un problema grande en varios más pequeños y sencillos. En el curso se utiliza Scratch que es uno de los lenguajes de programación más fáciles de usar, donde se pueden crear programas sobre animaciones, juegos e historias interactivas, moviendo y colocando bloques en forma coherente (Friss, 2016).

Pero ¡A Programar! es mucho más que una introducción a Scratch, ¡le enseña a un estudiante a pensar como programador, como ingeniero de software!, presenta los principios fundamentales de la computación, llevando a alumno a reflexionar en torno a temas tales como el diseño de algoritmos, la interacción con el usuario/a, la reutilización del código y el modelado de problemas. Aprendiendo todo esto a través de ejemplos prácticos detallados (que el mismo curso brinda o pide realizar) y entrevistas con personas expertas en su ámbito laboral, que mencionan la importancia de los conceptos de programación y como los aplican.

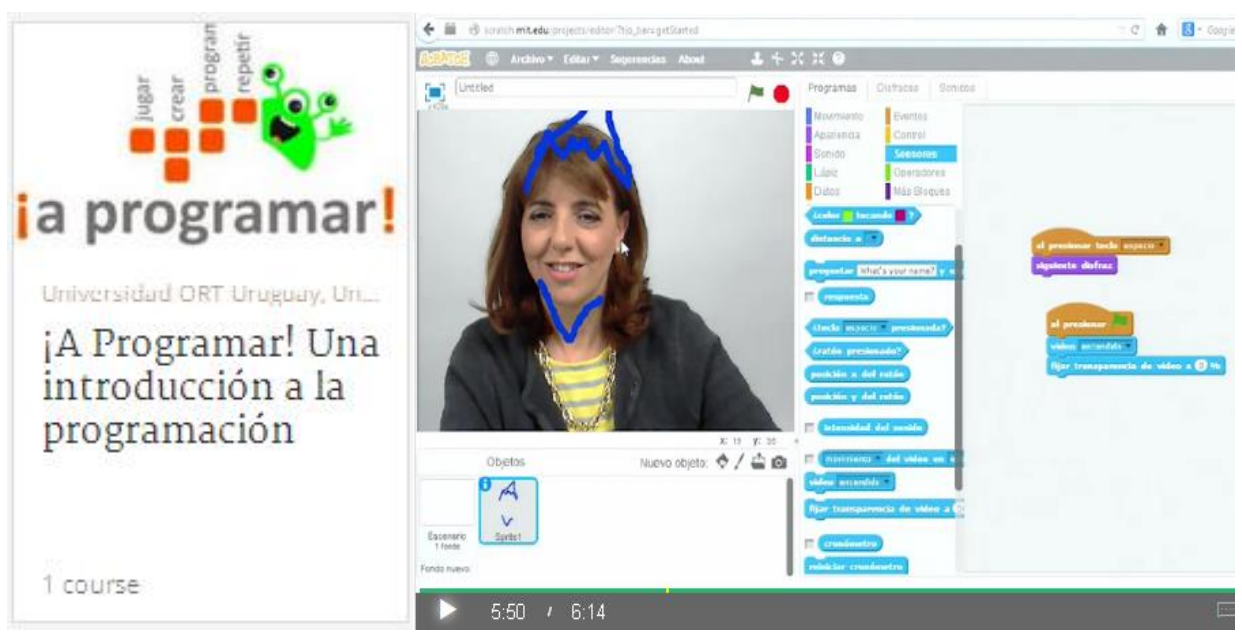

Figura 2. El curso XMOOC que se siguió en clase

$\overline{\text { Integración de un curso MOOC y de un PLN-PLE en un curso presencial sobre fundamentos de la }}$ programación. Joel Ricardo Jiménez Cruz. 
Además, en los lapsos entre videos se manejan divertidos cuestionarios y proyectos creativos. El curso propicia un sólido conocimiento de los fundamentos de la programación que permite avanzar hacia otros lenguajes o tomar cursos más avanzados.

El curso se da en 6 etapas; una breve introducción al curso y 5 temas con un periodo de tiempo de un poco más de dos meses. Estas etapas se revisaron por semana en el curso presencial y su división consiste en:

Introducción: En esta etapa se describe la dinámica del curso, las reglas, los objetivos y se da una breve descripción de lo que se hace en el curso.

Semana 1: Aquí se ven algunos conceptos básicos de programación; algoritmo, estructuras de control y representación de algoritmos. También se introduce el lenguaje Scratch (que es, algunos ejemplos introductorios, sus componentes y las opciones para trabajar) y otros conceptos como estructuras de decisión e iteración y pseudocódigo.

Semana 2: En esta semana se analiza la iteración controlada por una cantidad, el uso del "lápiz" en Scratch, un ejemplo al realizar una tarjeta de cumpleaños y la teoría inicial sobre el diseño de software. En esta semana se practica la descomposición de los algoritmos y la interacción con el usuario utilizando la cámara y el micrófono de la computadora.

Semana 3: En esta semana se consolidan algunos ejercicios realizados anteriormente, incluyendo el concepto de "variable". El aprendizaje central de esta semana consiste en darse cuenta de la importancia que tienen los algoritmos dentro de la programación.

Semana 4: En esta semana se ve la definición de procedimiento, y como éste ayuda a poder realizar un algoritmo más complejo. De igual forma se menciona la importancia de reutilizar los códigos ya realizados y como trabajan varios procedimientos dentro de un programa.

Semana 5: En esta semana se ocupan todos los pasos de ingeniería de software (análisis de requerimiento, especificación, diseño y arquitectura, programación, prueba, documentación y mantenimiento) para crear un divertido video juego (con base en el juego tradicional plantas contra zombis). También se hace mención y se enseña a utilizar el envío de mensajes ("broadcasting") y su concurrencia.

Al final de cada semana los alumnos se autoevalúan tomando una prueba automática que genera la plataforma de Coursera. En la figura 3 se pueden observar las calificaciones obtenidas por una alumna. 


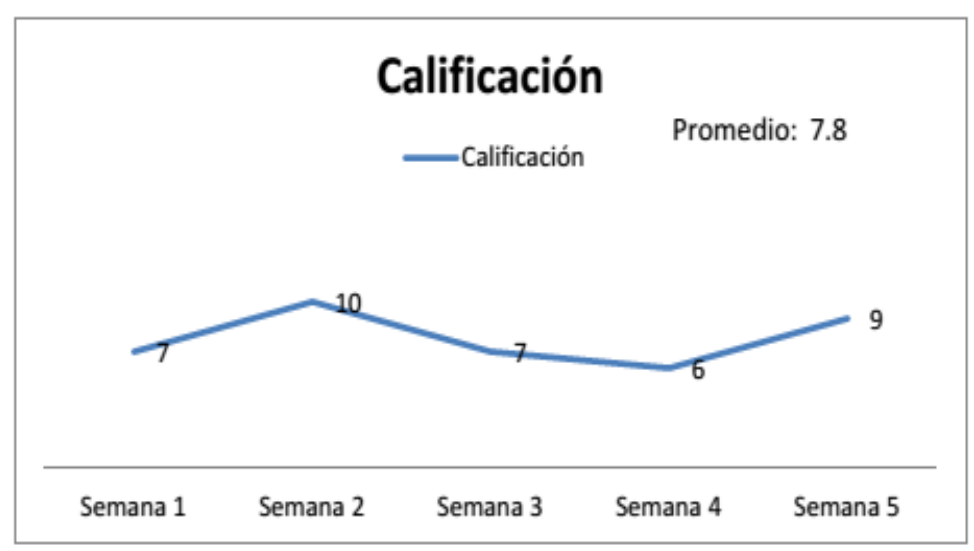

Figura 3. Calificaciones obtenidas por una alumna en el curso MOOC

El estudio y práctica en cada una de las semanas del curso MOOC se van sincronizando con los ejercicios y la teoría que se van revisando en el salón de clases.

\section{Metodología}

La metodología que se ha seguido en clases está sustentada por la del aprendizaje basado en proyectos que le permite al profesor y a los alumnos tener una visión y un objetivo bien planteados desde el principio del ciclo escolar (Jiménez, 2014). El marco pedagógico que soporta a los elementos presenciales y virtuales se da dentro de un contexto de aprendizaje conectivista que pretende alcanzar diferentes niveles de interacción e involucramiento cognitivo entre los participantes (Wang, Chen, Anderson, 2014). También en la dinámica de las clases se utiliza la técnica de la clase invertida para complementar lo que se ha visto en los videos y actividades del curso MOOC.

En la técnica de la clase o aula invertida los estudiantes revisan en casa videos cortos o lecciones multimedia hechos por el profesor del curso o por otros, antes de la siguiente clase y durante la clase los estudiantes junto con el profesor discuten dudas, aprendizajes y otros aspectos relevantes de los videos. Esta técnica cambia el aprendizaje directo a un espacio más individualizado con ayuda de la tecnología para privilegiar el aprendizaje activo, la instrucción por pares y la atención a diversos estilos de aprendizaje. El trabajo fuera del aula funciona como un entrenamiento previo que capacita a los estudiantes para procesar la información de una manera más eficiente (Salinas et al, 2015).

En la clase presencial se revisa la metodología del aprendizaje de los lenguajes de programación a través del pseudocódigo y los diagramas de flujo con el fin de que los alumnos obtengan las habilidades y competencias para trabajar en distintos lenguajes de programación.

En la figura 4 se muestran los objetivos señalados en el EVEA (http://virtuami.izt.uam.mx/aulas/apresencial/course/index.php?categoryid=5) que se ha implementado en Moodle con el fin de coordinar todos los elementos del ecosistema híbrido y facilitar la comunicación y disponibilidad del profesor.

Integración de un curso MOOC y de un PLN-PLE en un curso presencial sobre fundamentos de la programación. Joel Ricardo Jiménez Cruz. 

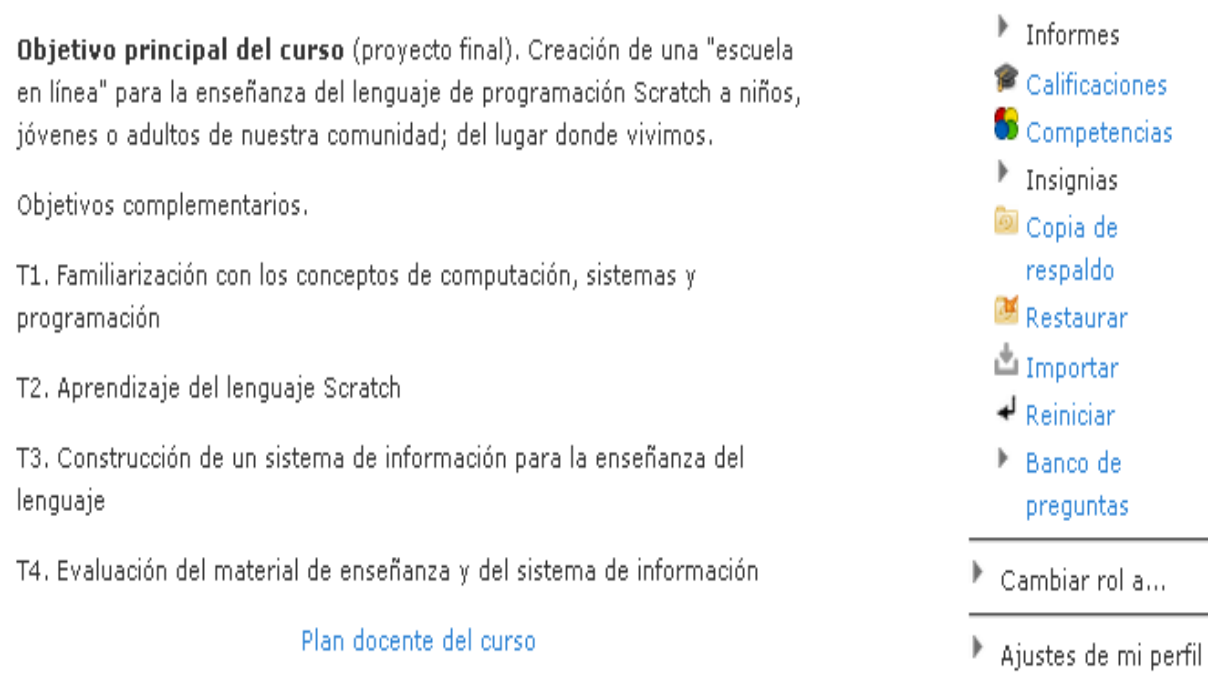

Figura 4. Coordinación de los componentes virtuales y presenciales en Moodle

Los temas vistos por semana en Coursera se retroalimentan en clase, en donde se revisan y detallan definiciones y conceptos de manera más amplia para complementar lo aprendido. También se elaboran ejemplos de algoritmos considerando las fases de análisis, diseño y realizando pruebas de escritorio para verificar su funcionamiento correcto.

\section{Resultados}

Con la combinación de los métodos clásicos de la enseñanza presencial y los métodos novedosos del aprendizaje virtual que se han mencionado en las secciones anteriores, se desarrollaron habilidades necesarias en cada una de estas modalidades educativas. En la parte virtual, se desarrollaron habilidades digitales relacionadas con la teoría y práctica de los PLE-PLN y el uso del EVEA. En la parte presencial se puso énfasis en desarrollar principalmente el pensamiento informático y computacional.

A continuación se hace una relatoría de los resultados obtenidos a través de un análisis cualitativo de los avances observados, de los reportes realizados y de los blogs y sitios construidos por los alumnos durante el curso.

Para el diseño del sitio web de la "escuela en línea", los estudiantes consideraron aspectos técnicos, estéticos, didácticos y psicopedagógicos, entre ellos; el tipo de audiencia (niños, jóvenes, adultos), la utilización de juegos, retos, animaciones que motivan al usuario, con la incorporación de programas, ejercicios, cuestionarios, ejemplos, exposiciones y concursos. Se señaló la diferencia entre diferentes tipos de instrucción teórica o práctica y el uso de videoconferencias o videos acompañados de retroalimentación, así como la realización de evaluaciones periódicas.

Un diagrama de navegación representativo del sitio web desarrollado por un alumno se muestra en la figura 5 .

Integración de un curso MOOC y de un PLN-PLE en un curso presencial sobre fundamentos de la programación. Joel Ricardo Jiménez Cruz.

Página 10 de 17 


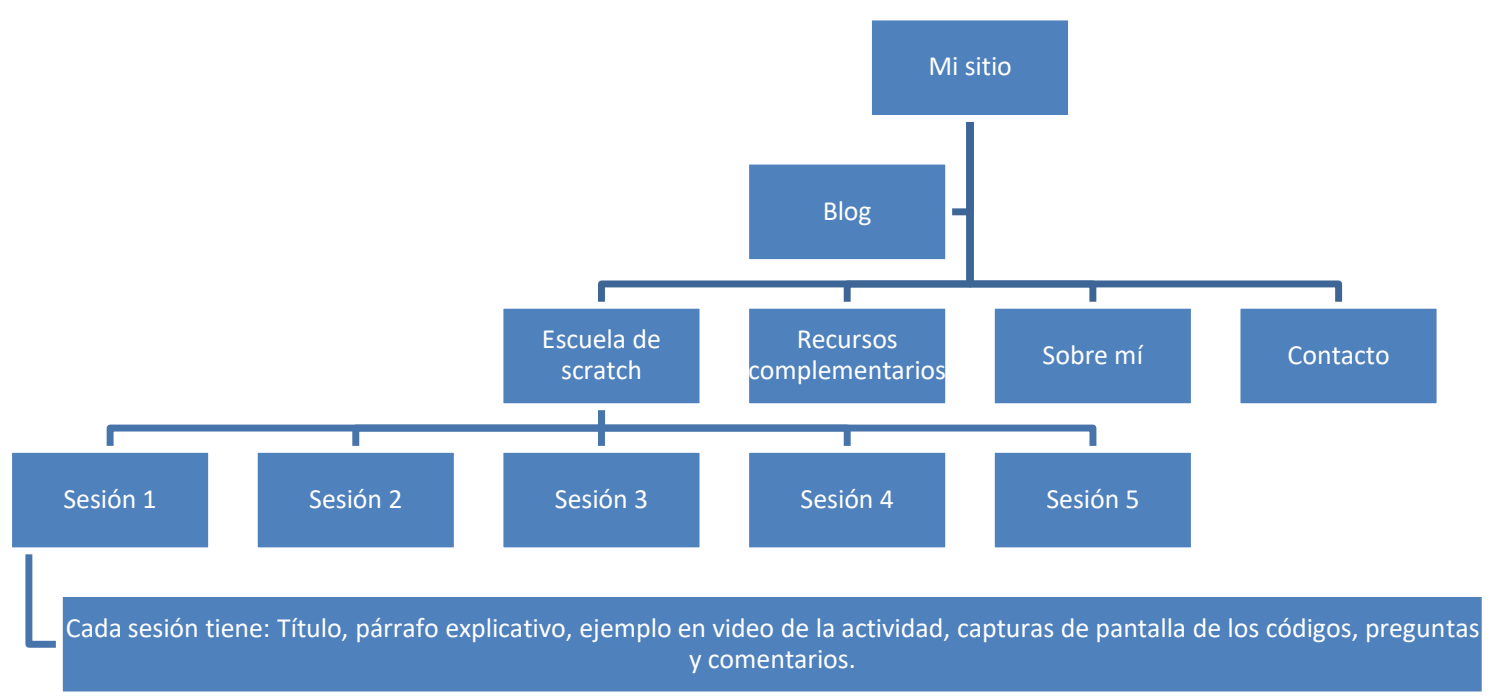

Figura 5. Diagrama de navegación para la implementación del sitio web

En la figura 6 y como muestra se despliegan algunos sitios desarrollados por los alumnos.

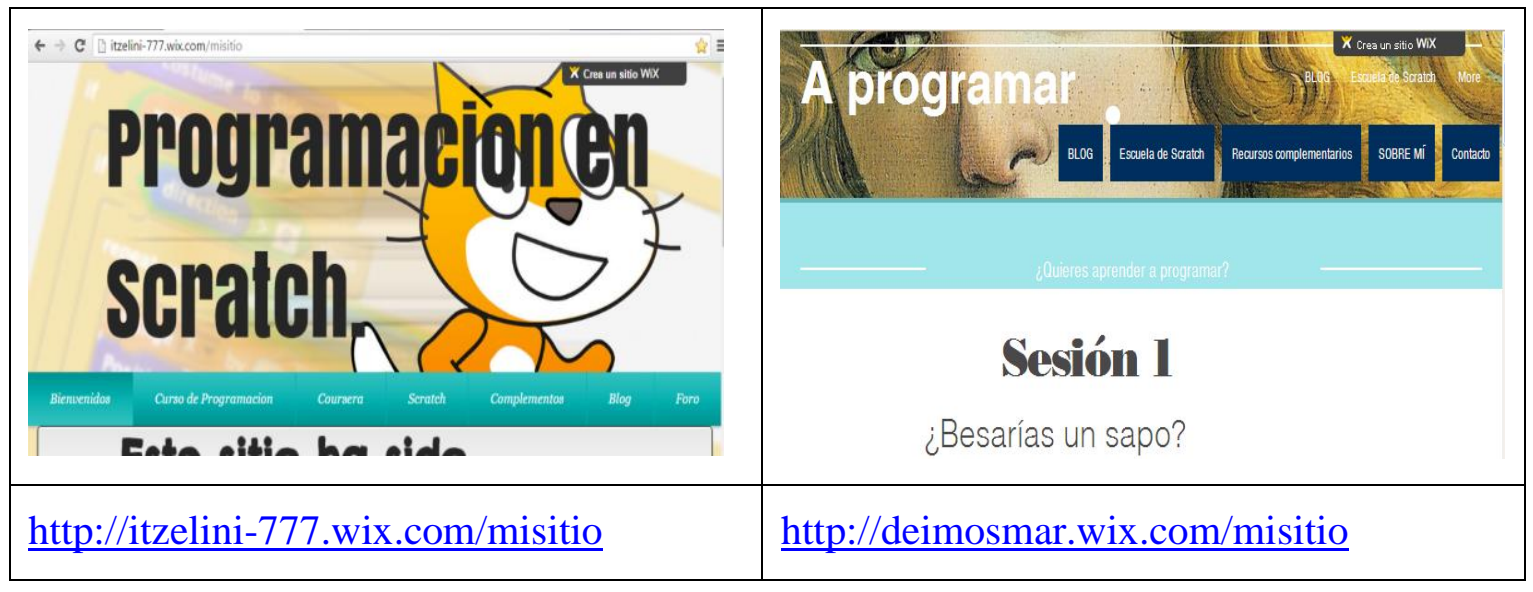

Figura 6. Algunos ejemplos de sitios web desarrollados por los alumnos

Respecto a la experiencia que obtuvieron los alumnos, en la figura 7 se muestran algunos ejercicios del sitio web de una alumna. A continuación se reescribe su impresión del curso:

"El curso de Scratch y hacer el sitio de la escuela de este lenguaje de programación fueron muy enriquecedores, incluso divertidos. Scratch tiene una interfaz muy amigable, apta para personas sin ningún conocimiento previo. Antes creía que hacer páginas web era muy complicado y tedioso, ahora sé que con WIX resulta sencillo e intuitivo (gracias a dios), y que uno lo puede hacer sin ayuda. Sólo se necesita tiempo y dedicación (y el tiempo precisamente no abunda en estos días). Realicé el diseño de la escuela de Scratch por sesiones siguiendo las semanas de Coursera para aprovecharme

Integración de un curso MOOC y de un PLN-PLE en un curso presencial sobre fundamentos de la programación. Joel Ricardo Jiménez Cruz. 
de lo ya hecho y que me parecía bien hecho. De esta manera podía retomar del curso el aumento progresivo en la complejidad de los ejercicios".

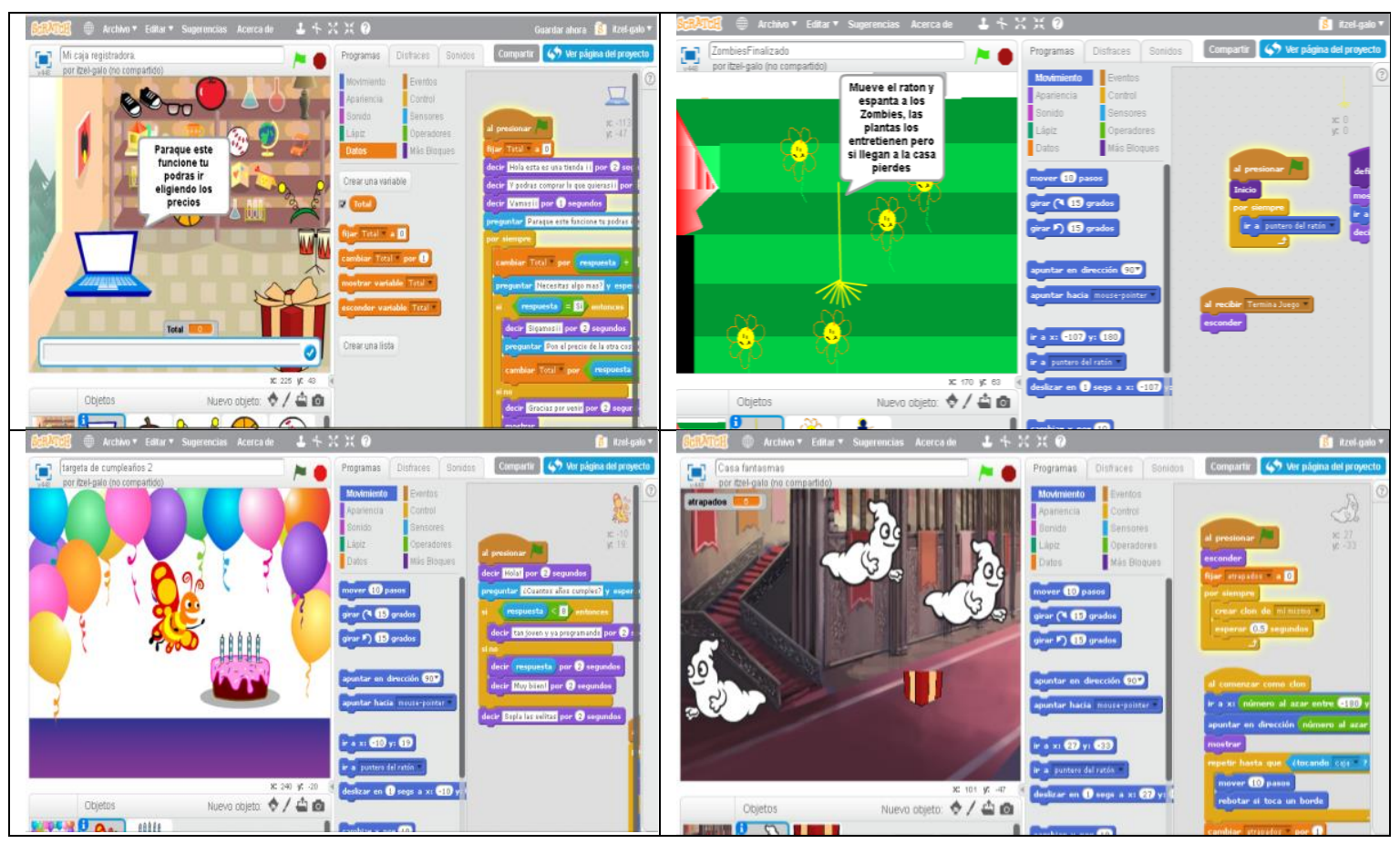

Figura 7. Ejercicios de Scratch del sitio http://itzelini-777.wix.com/misitio

Otra alumna menciona:

"Para terminar el curso cada uno de los compañeros tuvimos que presentar nuestras páginas, y el profesor realizó una pregunta, “¿qué si esta clase nos brindaba algún apoyo para nuestra licenciatura?" y en realidad, sí. Creó que esto nos ayudó a comprender el funcionamiento de las computadoras, de los celulares y demás cosas tecnológicas que aunque son muy diferentes en el aspecto físico, por dentro, es decir, en su funcionamiento tienen los mismos sistemas programados, para realizar tan solo un objetivo, realizar las órdenes que nosotros damos. Las personas tenemos que aprender a utilizar las máquina, tan solo por lo que son ya que muchos dan valor, como si los objetos tuvieran vida propia, o como si estos pensaran por sí mismos y en realidad no. Nosotros los humanos somos quienes le brindamos esa inteligencia artificial, nuestra inteligencia es la capacidad de ellos".

En cuanto al desarrollo de una red de aprendizaje social para formar el PLN, en la figura 8 se muestran algunos comentarios realizados por los compañeros del grupo al blog de un estudiante (http://deimosmar.wixsite.com/misitio/blog/page/1).

Integración de un curso MOOC y de un PLN-PLE en un curso presencial sobre fundamentos de la programación. Joel Ricardo Jiménez Cruz. 


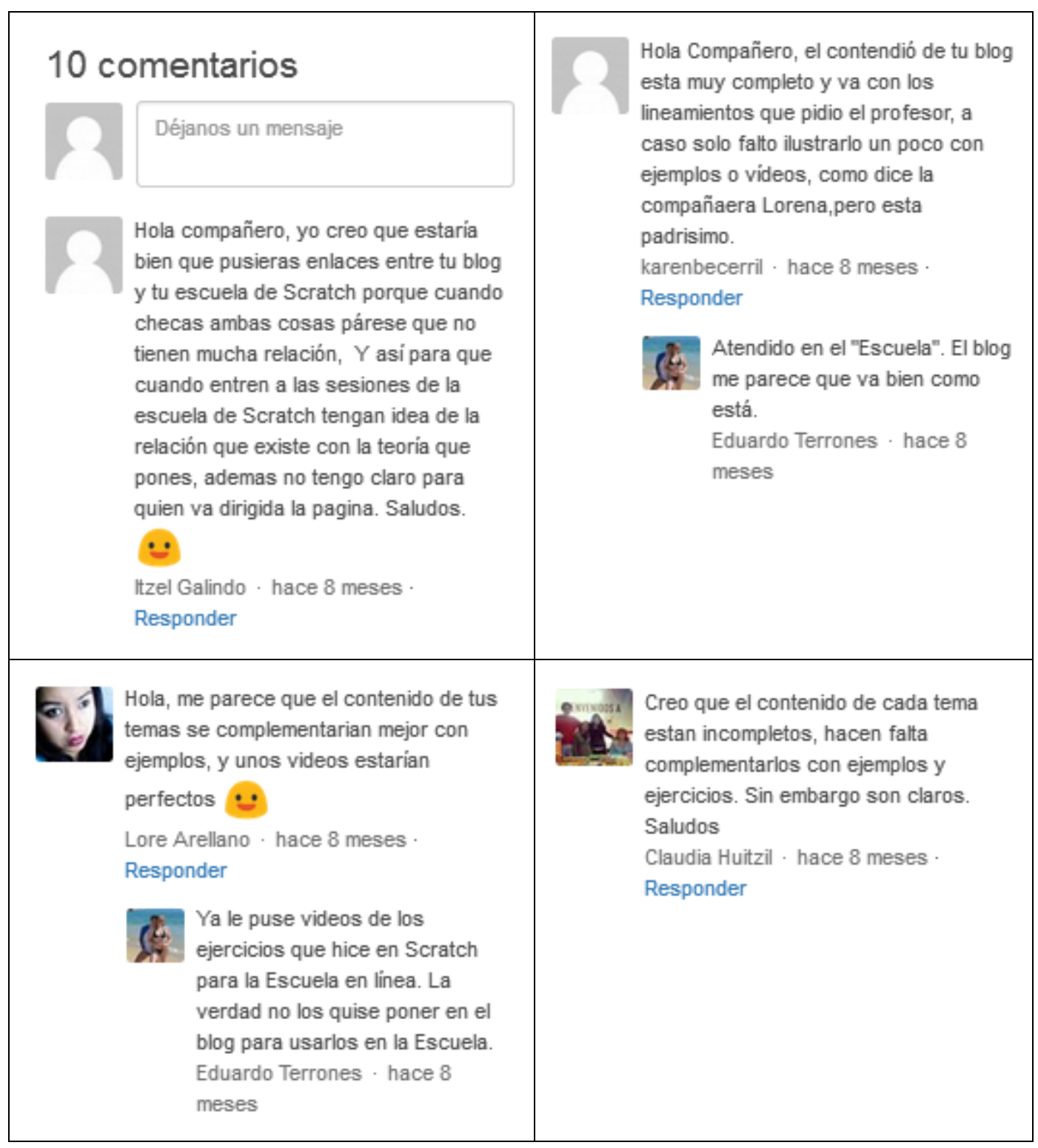

Figura 8. Algunos comentarios de los compañeros hacia el blog de un estudiante

\section{Comentarios y conclusiones}

La educación híbrida (b-learning) es un ejemplo de innovación educativa en crecimiento, que es necesario investigar, difundir, apoyar y desarrollar. Inicialmente los cursos MOOCs se diseñaron como cursos independientes, pero ahora algunas materias del aprendizaje híbrido han empezado a utilizar sus beneficios. Los PLE-PLN son instrumentos para la construcción paulatina y propia del conocimiento. Los EVEAs son útiles para la coordinación, integración y sincronización de los elementos virtuales y presenciales.

La aportación del modelo híbrido con estos elementos es prometedora. La combinación de los elementos procura optimizar y potenciar los procesos de enseñanza-aprendizaje al tener una mayor flexibilidad en el tiempo de aprendizaje, en la adquisición de competencias analógicas y digitales, en el desarrollo de un aprendizaje autónomo y

Integración de un curso MOOC y de un PLN-PLE en un curso presencial sobre fundamentos de la programación. Joel Ricardo Jiménez Cruz. 
significativo, en un seguimiento del progreso del aprendizaje y en un acceso pronto a los recursos educativos y a la interacción y comunicación de los participantes. Por otro lado, se requiere de un proceso paulatino de formación, adaptación, manejo de los recursos y familiarización en esta metodología presencial-virtual, tanto por parte del profesor como de los estudiantes, así como también de las instituciones educativas (Moral y Fernández, 2014).

Los PLE-PLN y los cursos masivos representan una evolución de la educación abierta que se basan en una concepción conectivista, donde la creación y distribución del conocimiento emerge como resultado de los intercambios de información que se dan entre los nodos de la red. Estos entornos de aprendizaje abiertos, gratuitos y democráticos han supuesto la posibilidad de que miles de personas de todas las edades y de todo el mundo tengan acceso a la educación. Tienen una gran variedad de recursos que fomentan el interés y participación, mejorando las habilidades sociales y comunicativas, a la vez que permite adquirir destrezas para el aprendizaje permanente.

El mejoramiento de la eficacia en la inclusión de PLE-PLN y MOOCs en las clases presenciales reside en modelos como el que se ha presentado en este trabajo que consideran las tecnologías de la información, la comunicación, el aprendizaje, el conocimiento, el empoderamiento y la participación social de los implicados (Reig, 2012).

El EVEA ha funcionado como un centro coordinador de las actividades satelitales de los PLE-PLN de cada uno de los alumnos. Funciona como un apoyo didáctico pedagógico a las clases presenciales y al avance en el desarrollo de los elementos virtuales.

El ecosistema construido promueve la búsqueda, localización, gestión de información y generación de conocimiento que son útiles para los estudiantes que pasan de ser sólo consumidores pasivos en creadores de contenidos y de materiales. Estas habilidades digitales desarrolladas durante el curso han sido promovidas por el profesor en el salón de clases y se comprueban al observar que los alumnos han podido construir sus sitios web.

Los alumnos conocen muy bien los procedimientos y herramientas digitales para crear cuentas y utilizar diversas redes sociales como Facebook y Twitter lo cual ha permitido que la curva de aprendizaje sea menor en la incorporación y uso de las nuevas herramientas como la creación de su PLE-PLN en WIX y la familiarización con la metodología del curso MOOC, todo esto en beneficio de su aprendizaje en la materia de fundamentos de programación.

En los reportes de fin de curso que se revisaron, los estudiantes apreciaron las bondades del ecosistema utilizado. Por un lado la clase presencial les proporcionó la guía para un aprendizaje tutorado, los cursos MOOC les permitieron estudiar a su propio ritmo y complementar su conocimiento, descubriendo que ellos eran capaces de aprender por si mismos la programación de computadoras por medio del lenguaje Scratch. La construcción del PLE-PLN con la plataforma de WIX les permitió tomar confianza y conocer que la implementación era factible a pesar de que ellos no tenían antecedentes computacionales robustos.

Integración de un curso MOOC y de un PLN-PLE en un curso presencial sobre fundamentos de la programación. Joel Ricardo Jiménez Cruz. 
Existe una infinidad de plataformas para el desarrollo de sitios web, entre ellas Blogger y Wordpress, sin embargo la plataforma WIX que se utilizó en el curso permite crear los sitios con facilidad y con una presentación profesional excelente.

Como se muestra en la figura 8, se avanzó en el desarrollo de una red de aprendizaje social para formar el PLN de cada alumno; se realizaron comentarios entre los compañeros para mejorar el blog y el sitio web. Falta avanzar y madurar el desarrollo de una comunidad externa que realimenten la finalidad del blog y de la escuela en línea.

Se observó en los alumnos, a medida que iba avanzado el curso, una mayor autonomía y un mayor grado de habilidad para la solución de los problemas informáticos y computacionales relacionados con el aprendizaje del lenguaje Scratch y el desarrollo del proyecto de la escuela en línea. Esta observación también está documentada en Vilà, Rubio y Berlanga (2014) que realizan una investigación formativa a través del aprendizaje orientado a proyectos.

Una extensión de presente trabajo consistiría en investigar marcos de referencia para ambientes conectivistas como el que propone Wang et al (2014) en el cual se evalúa el desarrollo cognitivo y de aprendizaje en los estudiantes a través de cuatro niveles de interacción (que van desde el nivel más sencillo al más complejo); operativa, de búsqueda, significativa y de innovación.

Presentación del artículo: 14 de Febrero de 2017

Fecha de aprobación: 15 de marzo de 2017

Fecha de publicación: 31 de Marzo de 2017

Jiménez, J.R. (2017). Integración de un curso MOOC y de un PLN-PLE en un curso presencial sobre fundamentos de la programación. RED. Revista de Educación a Distancia, 53. Consultado el (dd/mm/aaaa) en http://www.um.es/ead/red/53/jimenez.pdf

\section{Bibliografía}

Best College Online (2013). Are MOOCs the Future of Online Education? http://www.bestcollegesonline.org/moocs/?utm_source=linkedin\&utm_medium=so cial\&utm_content=2952169?utm_source=linkedin\&utm_medium=social\&utm_con tent $=3075782$

Bralić A., Divjak B. (2016). Use of moocs in traditional classroom: blended learning approach. Proceedings of the 9th European Distance and E-Learning Network Research Workshop. Recuperado de http://www.eden-online.org/wpcontent/uploads/2016/10/BRPA-RW9_Bralic_Divjak.pdf

Integración de un curso MOOC y de un PLN-PLE en un curso presencial sobre fundamentos de la programación. Joel Ricardo Jiménez Cruz. 
Castaño, C., Garay, U., Maiz I. (2017). Factores de éxito académico en la integración de los MOOC en el aula universitaria. Revista española de pedagogía, $\mathrm{N}^{\circ} 266$, Recuperado de http://revistadepedagogia.org/index.php/es/295-vol-xllv-2017/n266-enero-abril-2017/1435-factores-de-exito-academico-en-la-integracion-de-losmooc-en-el-aula-universitaria

Crosslin, Matt (2016) "From Instructivism to Connectivism: Theoretical Underpinnings of MOOCs," Current Issues in Emerging eLearning: Vol. 3: Iss.1, Article 6. Recuperado de: http://scholarworks.umb.edu/ciee/vol3/iss1/6

Downes S. (2009). Learning Networks and Connective Knowledge. Recuperado de http://www.downes.ca/post/36031

Dunbar, R. (1993). Coevolution of neocortical size, group size and language in humans. In: Behavioral and Brain Sciences. 16 (4), p. 681-735. Recuperado de http://www.uvm.edu/ pdodds/files/papers/others/1993/dunbar1993a.pdf

Eshach, H. (2007). Bridging In-school and Out-of-school Learning: Formal, NonFormal, and Informal Education. J Sci Educ Technol 16: 171-190. Recuperado de https://www.researchgate.net/publication/225729733_Bridging_In-

school_and_Out-of-school_Learning_Formal_Non-

Formal_and_Informal_Education

Fernández, C. (2015). La importancia del diseño en los cursos MOOC. VI congreso virtual iberoamericano de calidad en educación virtual y a distancia, pp: 1-8. http://www.eduqa.net/eduqa2015/images/ponencias/eje1/1_p_FERNANDEZ_Clara _GARCIA_Noelia_La_importancia_del_diseno_en_los_cursos_MOOC.pdf

Friss de Kereki I. (2016). ¡A programar! Una introducción a la programación. Recuperado de https://www.coursera.org/learn/a-programar

Hansen, J., Reich, J. (2015). Democratizing education? Examining access and usage patterns in massive open online courses. Science, 04, Vol. 350, Issue 6265. Recuperado de http://science.sciencemag.org/content/350/6265/1245

Illich, I. (1985). La sociedad escolarizada. Recuperado de http://www.ivanillich.org.mx/desescolar.pdf

Jiménez, J. (2014). Modelo de diseño instruccional semipresencial basado en proyectos a partir de un LMS y PLEs. Integrando ambientes organizacionales y personales. RED, Revista de Educación a Distancia. Número 42. Número monográfico sobre "Experiencias y tendencias en affordances educativas de campus virtuales universitarios". Recuperado de http://www.um.es/ead/red/42/joel.pdf

Joseph, M. (2015). Effectiveness of Integrating MOOCs in Traditional Classrooms for Undergraduate Students. International Review of Research in Open and Distributed Learning. Volume 16, Number 5. Recuperado de http://www.irrodl.org/index.php/irrodl/article/view/2222/3437

Lane, L. M. (2012, August 15). Three kinds of MOOCs. Recuperado de http://www.lisahistory.net/wordpress/2012/08/three-kinds-of-moocs/

Integración de un curso MOOC y de un PLN-PLE en un curso presencial sobre fundamentos de la programación. Joel Ricardo Jiménez Cruz. 
Marín V., Negre F., Pérez A. (2014). Entornos y redes personales de aprendizaje (PLEPLN) para el aprendizaje colaborativo. Revista Comunicar $N^{\circ}$ 42: págs. 35-43. Recuperado de http://www.revistacomunicar.com/indice/articulo.php?numero=422014-03

Moral, M., Fernández M. (2014). El b-learning como instrumento para la adquisición de competencias en el marco del espacio europeo de educación superior (EEES): una propuesta docente. Proceedings del X Foro Internacional sobre Evaluación de la Calidad de la Investigación y la Educación Superior, pp. 19-22. Recuperado de http://www.ugr.es/ aepc/FECIES_13/PROCEEDING/5.pdf

Plourde, M. (2013). MOOC Poster V(3). Recuperado de https://www.flickr.com/photos/mathplourde/8620174342

Reig, D. (2012). Aprendizaje y evolución de lo tecno-social. Recuperado de http://www.dreig.eu/caparazon/2012/03/07/aprendizaje-tecnosocial/

Rheingold, H. (2010). Attention, and Other 21st-Century Social Media Literacies. Educause Review, v45 n5 p14-24. Recuperado de http://er.educause.edu/articles/2010/10/attention-and-other-21stcentury-socialmedia-literacies

Salinas, P., Quintero, E., Rodríguez, J. (2015). Curso híbrido y de aula invertida apoyado en MOOC: experiencia de autoevaluación. Revista de Innovación $\begin{array}{lllll}\text { Educativa, } & \text { Volumen } & 7 & \text { (1). }\end{array}$ http://www.udgvirtual.udg.mx/apertura/index.php/apertura/article/view/608/pdf

Scharmer, O. (2015). MOOC 4.0: The Next Revolution in Learning \& Leadership. Recuperado de http://www.huffingtonpost.com/otto-scharmer/mooc-40-the-nextrevoluti_b_7209606.html

Siemens, G. (2004). Conectivismo: Una teoría de aprendizaje para la era digital. Recuperado de https://es.scribd.com/doc/201419/Conectivismo-una-teoria-delaprendizaje-para-la-era-digital

Tathagata R., Malapati, A., Murthy B. (2016). Teaching Computer Programming Using MOOCs in Multiple Campuses: Challenges and Solutions. IEEE Eighth International Conference on Technology for Education (T4E). Pages: 160 - 163. Recuperado de http://ieeexplore.ieee.org/abstract/document/7814816/

Vilà, R. Rubio, M. Berlanga, V. (2014). La investigación formativa a través del aprendizaje orientado a proyectos: una propuesta de innovación en el grado de pedagogía. Innovación Educativa, $\mathrm{n}^{\mathrm{o}}$ 24: pp. 241-258. http://www.usc.es/revistas/index.php/ie/article/view/1586/2404

Wang, Z., Chen, L., \& Anderson, T. (2014). A framework for interaction and cognitive engagement in connectivist learning contexts. The International Review of Research in Open and Distributed Learning, 15(2). Recuperado de http://www.irrodl.org/index.php/irrodl/article/view/1709/2838

Integración de un curso MOOC y de un PLN-PLE en un curso presencial sobre fundamentos de la programación. Joel Ricardo Jiménez Cruz. 